\title{
Risk factors for Veteran food insecurity: findings from a National US Department of Veterans Affairs Food Insecurity Screener
}

\author{
Alicia J Cohen ${ }^{1,2,3, *}$ (1), David M Dosa ${ }^{1,3,4}$, James L Rudolph ${ }^{1,3,4}$, \\ Christopher W Halladay', Michele Heisler ${ }^{5,6,7}$ and Kali S Thomas ${ }^{1,3}$ \\ ${ }^{1}$ Center of Innovation in Long Term Services and Supports, VA Providence Healthcare System, Providence, RI, USA: \\ ${ }^{2}$ Department of Family Medicine, Alpert Medical School of Brown University, Providence, RI, USA: ${ }^{3}$ Department of \\ Health Services, Policy, and Practice, Brown University School of Public Health, Providence, RI 02903, USA: \\ ${ }^{4}$ Department of Medicine, Alpert Medical School of Brown University, Providence, RI, USA: ${ }^{5}$ Center for Clinical \\ Management Research, VA Ann Arbor Healthcare System, Ann Arbor, MI, USA: ${ }^{6}$ Department of Internal Medicine, \\ University of Michigan Medical School, Ann Arbor, Michigan: ${ }^{7}$ Department of Health Behavior and Health Education, \\ University of Michigan School of Public Health, Ann Arbor, MI, USA
}

Submitted 21 December 2020: Final revision received 19 October 2021: Accepted 2 November 2021: First published online 8 November 2021

\begin{abstract}
Objective: Food insecurity is associated with numerous adverse health outcomes. The US Veterans Health Administration (VHA) began universal food insecurity screening in 2017. This study examined prevalence and correlates of food insecurity among Veterans screened.

Design: Retrospective cross-sectional study using VHA administrative data. Multivariable logistic regression models were estimated to identify sociodemographic and medical characteristics associated with a positive food insecurity screen.

Setting: All US Veterans Administration (VA) medical centres ( $n$ 161).

Participants: All Veterans were screened for food insecurity since screening initiation (July 2017-December 2018).

Results: Of 3304702 Veterans screened for food insecurity, 44298 were positive on their initial screen ( $1.3 \%$ of men; $2.0 \%$ of women). Food insecurity was associated with identifying as non-Hispanic Black or Hispanic. Veterans who were non-married/partnered, low-income Veterans without VA disability-related compensation and those with housing instability had higher odds of food insecurity, as did Veterans with a BMI $<18 \cdot 5$, diabetes, depression and post-traumatic stress disorder. Prior military sexual trauma (MST) was associated with food insecurity among both men and women. Women screening positive, however, were eight times more likely than men to have experienced MST (48.9\% v. 5.9\%).

Conclusions: Food insecurity was associated with medical and trauma-related comorbidities as well as unmet social needs including housing instability. Additionally, Veterans of colour and women were at higher risk for food insecurity. Findings can inform development of tailored interventions to address food insecurity such as more frequent screening among high-risk populations, onsite support applying for federal food assistance programs and formal partnerships with community-based resources.
\end{abstract}

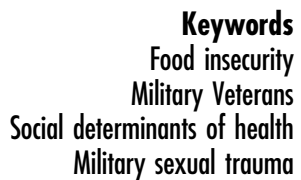

Keywords Food insecurity
Military Veterans Military sexual trauma
Food insecurity — defined as limited or uncertain availability of nutritionally adequate and safe food ${ }^{(1)}$ — is associated with a range of adverse health outcomes ${ }^{(2,3)}$. Food insecurity is also associated with delayed or missed care ${ }^{(4,5)}$, increased acute care utilisation ${ }^{(4,6,7)}$ and higher healthcare costs $^{(6,8)}$. In 2020, $10 \cdot 5 \%$ of US households-and $14.8 \%$ of households with children-reported being food insecure at least some time during the prior 12 months $^{(9)}$. This financial strain is often more pronounced in households with individuals with acute or chronic medical conditions ${ }^{(10-12)}$. Levels of food insecurity increased dramatically since the start of the COVID-19 pandemic, particularly among households with children and among racial and ethnic minority groups, due to a variety of factors including 
employer shut downs, loss of childcare and in-person schooling and health-related economic hardship ${ }^{(9,13-15)}$.

Limited research has focused on food insecurity among the $8 \%$ of the US population that has served in the US armed forces. Many Veterans face unique economic and employment challenges following their military service, stemming both from service-related mental and physical health issues as well difficulty reintegrating into civilian life ${ }^{(16)}$. Previously published estimates of the prevalence of food insecurity among US Veterans vary from a low of $6 \%$ to as high as $24 \%$ depending on the criteria utilised $^{(3,16-20)}$. Two studies have examined food insecurity among the general Veteran population using nationally representative survey data, with respective prevalence rates of $6.5 \%$ in the prior $30 \mathrm{~d}^{(17)}$ and $8.4 \%$ in the prior 12 months $^{(18)}$. A study analysing data from 2002 to 2008 waves of the Veterans Aging Cohort Study, a multisite investigation of Veterans receiving Department of Veterans Affairs (VA) health care, found that $24 \%$ of Veterans reported food insecurity ${ }^{(3)}$. Higher rates of food insecurity have been reported among Veterans of Iraq and Afghanistan $(27 \%)^{(21)}$, women Veterans $(28 \%)^{(5)}$, homeless and formerly homeless Veterans $(49 \%)^{(22)}$ and Veterans with serious mental illness $(35 \%)^{(17)}$.

The Veterans Health Administration (VHA), the country's largest integrated federally funded health care system, has invested heavily in screening for and addressing Veteran food insecurity. Following 2015 congressional briefings on Veteran food insecurity, recommendations from a subsequently chartered VA Ensuring Veteran Food Security Workgroup, and a food insecurity screening pilot in VA homeless clinics ${ }^{(22)}$, VHA developed a national food insecurity screener in 2017 . This food insecurity clinical reminder, which is integrated into the electronic health record, prompts staff to administer a single-item screening question annually to all Veterans receiving VA health care who are not residents of a long-term care facility. Veterans endorsing food insecurity based on the single-item screener are offered a referral to a social worker and/or a dietitian as part of the clinical reminder. The food insecurity clinical reminder was first piloted at six sites across the country in July 2017, and implemented across VA medical centres nationally in October $2017^{(23)}$.

Little is known about prevalence or correlates of food insecurity among Veterans receiving VHA care, as measured by this universal VA food insecurity screener. To address this gap, we analysed data from the first 18 months following implementation of the food insecurity clinical reminder to identify: (1) the prevalence of reported food insecurity and (2) selected sociodemographic, medical and psychosocial characteristics associated with a positive screen. Based on prior literature, we hypothesised that while many sociodemographic and medical characteristics associated with food insecurity among US Veterans would be similar to those in the general US population, US Veterans may have additional military service and/or trauma-related risk factors for food insecurity.

\section{Methods}

\section{Data source and study cobort}

Data were extracted from the VA Corporate Data Warehouse, a national data repository that includes VA administrative and electronic health record data. The cohort consisted of all Veterans who were screened using the VA food insecurity clinical reminder between when it was first piloted in July 1, 2017 and December 31, 2018. Veterans were eligible for screening with the clinical reminder if they (1) received VA clinical care during the above time period, and (2) were not residing in a long-term care facility. During this time period, 3513321 food insecurity clinical reminder screens were completed. An additional $7496(0 \cdot 2 \%)$ of Veterans declined or were unable to answer the screening question, or were flagged in the electronic health record to receive the food insecurity clinical reminder but were ineligible due to residence in a longterm care facility. For those Veterans screened more than once during the study period, we restricted analyses to their first food insecurity screen, resulting in a final analytic sample of 3304702 Veterans.

\section{Measures}

The food insecurity clinical reminder prompts VA clinical staff to ask each eligible Veteran: 'In the past 3 months did you ever run out of food and were you not able to access more food or have the money to buy more food?' (yes/no). A 'yes' response is considered a positive screen for food insecurity.

All clinical reminder data are housed in the Corporate Data Warehouse in a national 'Health Factors' database. Sociodemographic characteristics-including gender, age at the time of screening, race, ethnicity, marital status, history of military sexual trauma (MST) and Veteran enrolment priority status-were obtained from the medical record. MST is defined as sexual assault or harassment experienced during military service, and is a standardised screen administered to all Veterans. Enrolment priority determines Veterans' eligibility for, and cost-share associated with, VA health benefits. We collapsed enrolment priority into three categories based on VA benefits: Veterans with some percentage of service-connected disability and disabilityrelated VA compensation, Veterans not receiving VA disability compensation who are low income, and Veterans not receiving VA disability compensation who have income above the VA administered means test.

Most recent BMI was obtained from electronic health record vital sign data. We used the 10th revision of the International Classification of Diseases -10 codes to define medical and behavioural diagnoses including diabetes 
mellitus type II, hypertension, depression, post-traumatic stress disorder (PTSD) and substance use disorder. Separate VA clinical reminder data were used to define current tobacco use in the prior year. We defined homelessness/housing instability as having a positive homelessness clinical reminder screen and/or an International Classification of Diseases-10 code associated with homelessness or housing instability in the prior year. We identified Veterans living in a rural area using a standardised VA definition based on rural-urban commuting area codes.

\section{Statistical methods}

Standard descriptive statistics (frequencies and percentages) were calculated for all variables. We estimated bivariate and multivariable logistic regression models to identify sociodemographic, medical and psychosocial characteristics associated with a positive food insecurity clinical reminder screen. Because of potential unobserved facility-level differences in screening practices and populations served, all models include fixed effects for VA medical centres. For covariates with $\geq 5 \%$ missing values_race/ethnicity, marital status and BMI—we included missing as a separate category in the regression models. Missing values for the remaining variables were each $\leq 1 \%$. All analyses were conducted both for the full sample and stratified by gender.

In order to account for potential differences in screening practices during the initial 'ramp up' of the clinical reminder as sites were adapting to a new screening instrument, we conducted sensitivity analyses excluding the first 6 months of screening data and restricting our sample to Veterans screened between January 2018 and December 2018. R, version 3.6.1, was used for all analyses. This study was approved by the Providence VA Medical Center Institutional Review Board

\section{Results}

Between July 2017 and December 2018, 3304702 Veterans were screened using the food insecurity clinical reminder (Table 1). Of those screened, 3005797 (91.0\%) were male and 298905 (9.0\%) were female. Female Veterans were overall younger than male Veterans. $43.6 \%$ of women and $26.5 \%$ of men identified as non-white. More than half of men (54.6\%) and nearly two-thirds of women (66.2\%) had some level of serviceconnected disability. Six percent of men and $8.2 \%$ of women had experienced homelessness or housing instability during the prior year. Clinically, the majority of men (73.2\%) and women (69.9\%) were overweight or obese. Men were more likely than women to have a diagnosis of diabetes (25.7\% v. 11.4\%) and hypertension (54.6\% and $28.0 \%$ ), whereas women were more likely to have a diagnosis of depression $(15.6 \% v \cdot 30.7 \%)$ and PTSD
( $14.2 \% v \cdot 21 \cdot 3 \%)$. Nearly one-third of women $(31 \cdot 1 \%)$ reported a history of MST, compared with $1.9 \%$ of men.

Overall, 44298 Veterans (1.3\%) screened positive for food insecurity on their initial clinical reminder screen ( $1.3 \%$ of men and $2.0 \%$ of women, Table 2 ). There was substantial variation in the percentage of positive screens across VA medical facilities, ranging from $0 \cdot 11$ to $11 \cdot 1 \%$ (median 1.2\%, IQR 0.9-1.6\%). Compared with Veterans screening negative for food insecurity, Veterans with a positive screen were more likely to be $<65$ years of age $(77.9 \% v .50 \cdot 2 \%)$, and to identify as a racial/ethnic minority ( $46 \cdot 3 \% v .27 \cdot 7 \%)$, non-married/partnered (70.2\% v. 41.7\%) and low income $(44.1 \% v \cdot 21.9 \%)$. Veterans with a positive food insecurity screen were also more likely have experienced homelessness or housing instability in the prior year $(58.1 \% v$. $5.5 \%)$; smoke tobacco (48.3\% v. 23.8\%); have a history of MST (11.6\% v. $4.4 \%)$ and have a diagnosis of depression (36.2\% v. 16.7\%), PTSD (23.8\%v. 14.7\%) and/or substance use disorder (28.9\% $v .8 .8 \%)$. Trends were overall similar in gender-stratified analyses (Table 2). The prevalence of MST was substantially higher among women regardless of food insecurity status-48.9\% of women with a positive food insecurity screen and $30.7 \%$ of women with a negative food insecurity screen reported a history of MST (compared with $6.0 \%$ and $1.8 \%$ of men, respectively).

Adjusted and unadjusted odd ratios for correlates of a positive food insecurity clinical reminder, stratified by gender, are presented in Table 3. In adjusted models specific to male Veterans, a positive food insecurity screen was associated with age $<65$ years (18-34 years: adjusted OR $(\mathrm{aOR})=1.93, \quad 95 \% \quad \mathrm{CI}=1.71, \quad 2.18 ; \quad 35-44 \quad$ years: $\mathrm{aOR}=1 \cdot 87,95 \% \mathrm{CI}=1 \cdot 68,2 \cdot 08 ; 45-54$ years: $\mathrm{aOR}=1 \cdot 66$, $95 \% \mathrm{CI}=1.51,1.82$; and 55-64 years: $\mathrm{aOR}=1.58,95 \%$ $\mathrm{CI}=1.44, \quad 1.73)$; identifying as non-Hispanic Black $(\mathrm{aOR}=1.32,95 \% \mathrm{CI}=1.25,1.40)$, Hispanic $(\mathrm{aOR}=1.48$, $95 \% \mathrm{CI}=1.06,2.08)$ or 'other' non-white, non-Hispanic race/ethnicity $(\mathrm{aOR}=1 \cdot 22,95 \% \mathrm{CI}=1 \cdot 12,1 \cdot 33)$, being non-married/partnered $(\mathrm{aOR}=1 \cdot 36,95 \% \mathrm{CI}=1 \cdot 30,1 \cdot 42)$, and having experienced homelessness or housing instability in the prior year $(\mathrm{aOR}=13 \cdot 79,95 \% \mathrm{CI}=12 \cdot 36,15 \cdot 40)$. Lowincome Veterans without service-connected disability compensation had higher odds of a positive food insecurity screen $(\mathrm{aOR}=1.52,95 \% \mathrm{CI}=1.47,1.58)$ relative to Veterans with service-connected disability compensation. Veterans who were clinically underweight (BMI $<18.5$, $\mathrm{aOR}=1 \cdot 31,95 \% \mathrm{CI}=1 \cdot 21,1 \cdot 41)$, had a diagnosis of diabetes $(\mathrm{aOR}=1 \cdot 13,95 \% \mathrm{CI}=1.09,1 \cdot 18)$, smoked tobacco ( $\mathrm{aOR}=1.35,95 \% \mathrm{CI}=1.30,1.41)$, had a diagnosis of depression or PTSD $(\mathrm{aOR}=1.25,95 \% \mathrm{CI}, 1.20,1.31$ and $\mathrm{aOR}=1 \cdot 05,95 \% \mathrm{CI}=1 \cdot 01,1 \cdot 09$, respectively) or a history of MST $(\mathrm{aOR}=1.42,95 \% \mathrm{CI}=1.35,1.50)$ also had increased odds of a positive screen. History of substance use disorder was associated with a positive clinical reminder screen in bivariate analyses, but not in the adjusted model.

Correlates of a positive clinical reminder were generally similar for women, with the exception that identifying as 
Table 1 Characteristics of US Veterans Health Administration patients screened using the food insecurity clinical reminder, overall and by gender: July 2017-December 2018

\begin{tabular}{|c|c|c|c|c|c|c|}
\hline \multirow{2}{*}{ Characteristics, $n(\%)$} & \multicolumn{2}{|c|}{$\begin{array}{l}\text { All Veterans } \\
\text { (n } 3304 \text { 702) }\end{array}$} & \multicolumn{2}{|c|}{$\begin{array}{l}\text { Male Veterans } \\
\text { (n } 3005797)\end{array}$} & \multicolumn{2}{|c|}{$\begin{array}{c}\text { Female Veterans } \\
(n 298 \text { 905) }\end{array}$} \\
\hline & $n$ & $\%$ & $n$ & $\%$ & $n$ & $\%$ \\
\hline \multicolumn{7}{|l|}{ Age } \\
\hline $18-34$ & 327916 & 9.9 & 262203 & $8 \cdot 7$ & 65713 & $22 \cdot 0$ \\
\hline $35-44$ & 309411 & 9.4 & 247201 & 8.2 & 62210 & $20 \cdot 8$ \\
\hline $45-54$ & 414583 & $12 \cdot 5$ & 350519 & 11.7 & 64064 & 21.4 \\
\hline $55-64$ & 616436 & $18 \cdot 7$ & 546335 & $18 \cdot 2$ & 70101 & 23.5 \\
\hline$\geq 65$ & 1636356 & 49.5 & 1599539 & 53.2 & 36817 & $12 \cdot 3$ \\
\hline \multicolumn{7}{|l|}{ Race } \\
\hline White, Non-Hispanic & 2198029 & $66 \cdot 5$ & 2043824 & $68 \cdot 0$ & 154205 & 51.6 \\
\hline Black, non-Hispanic & 573391 & $17 \cdot 4$ & 483020 & $16 \cdot 1$ & 90371 & $30 \cdot 2$ \\
\hline Hispanic & 216034 & 6.5 & 192561 & 6.4 & 23473 & 7.9 \\
\hline Other, non-Hispanic & 136791 & $4 \cdot 1$ & 120370 & 4.0 & 16421 & 5.5 \\
\hline Missing & 216559 & $6 \cdot 6$ & 197815 & $6 \cdot 6$ & 18744 & $6 \cdot 3$ \\
\hline \multicolumn{7}{|l|}{ Marital status } \\
\hline Married/partnered & 1749026 & $52 \cdot 9$ & 1643620 & 54.7 & 105406 & $35 \cdot 3$ \\
\hline Divorced/separated/widowed & 949157 & 28.7 & 835055 & $27 \cdot 8$ & 114102 & 38.2 \\
\hline Single, never married & 441903 & 13.4 & 374089 & $12 \cdot 4$ & 67814 & $22 \cdot 7$ \\
\hline Missing & 164616 & $5 \cdot 0$ & 153033 & $5 \cdot 1$ & 11583 & 3.9 \\
\hline Rural & 1106532 & 33.5 & 1031236 & 34.3 & 72296 & $25 \cdot 2$ \\
\hline \multicolumn{7}{|l|}{ Enrolment Priority* } \\
\hline SC Disabilityt & 1838974 & $55 \cdot 6$ & 1641146 & $54 \cdot 6$ & 197828 & $66 \cdot 2$ \\
\hline Non-SC and low-income $\ddagger$ & 735168 & $22 \cdot 2$ & 677176 & 22.5 & 57992 & $19 \cdot 4$ \\
\hline Non-SC and not low-income§ & 719751 & $21 \cdot 8$ & 678616 & $22 \cdot 6$ & 41135 & $13 \cdot 8$ \\
\hline Missing & 10809 & 0.3 & 8859 & 0.3 & 1950 & 0.7 \\
\hline Substance use disorder & 299230 & $9 \cdot 1$ & 279239 & $9 \cdot 3$ & 19991 & $6 \cdot 7$ \\
\hline Current smoker & 796003 & $24 \cdot 1$ & 734826 & 24.4 & 61177 & 20.5 \\
\hline \multicolumn{7}{|l|}{ BMI } \\
\hline$<18.5$ & 26442 & 0.8 & 23219 & 0.8 & 3223 & $1 \cdot 1$ \\
\hline $18 \cdot 5-24.9$ & 552761 & $16 \cdot 7$ & 492977 & $16 \cdot 4$ & 59784 & $20 \cdot 0$ \\
\hline $25-29 \cdot 9$ & 1076081 & $32 \cdot 6$ & 994038 & 33.1 & 82043 & $27 \cdot 4$ \\
\hline $30-34.9$ & 804549 & $24 \cdot 3$ & 735973 & 24.5 & 68576 & $22 \cdot 9$ \\
\hline$\geq 35$ & 528637 & $16 \cdot 0$ & 470119 & $15 \cdot 6$ & 58518 & $19 \cdot 6$ \\
\hline Missing & 316232 & $9 \cdot 6$ & 289471 & $9 \cdot 6$ & 26761 & $9 \cdot 0$ \\
\hline Diabetes & 807910 & 24.4 & 773693 & $25 \cdot 7$ & 34217 & 11.4 \\
\hline Hypertension & 1725228 & $52 \cdot 2$ & 1641425 & $54 \cdot 6$ & 83803 & 28.0 \\
\hline Depression & 561245 & $17 \cdot 0$ & 469471 & $15 \cdot 6$ & 91774 & $30 \cdot 7$ \\
\hline Post-traumatic stress disorder & 489391 & $14 \cdot 8$ & 425617 & $14 \cdot 2$ & 63774 & $21 \cdot 3$ \\
\hline History of military sexual trauma & 148677 & 4.5 & 55736 & 1.9 & 92941 & $31 \cdot 1$ \\
\hline
\end{tabular}

Abbreviation: SC, service connected; VA, US Department of Veterans Affairs; BMI, body mass index.

*Enrolment priority determines US Veterans' eligibility for, and cost-share associated with, VA health benefits.

†SC disability provides a monetary benefit paid to Veterans who are determined by VA to be disabled by an injury or illness that was incurred or aggravated during active military service.

$\ddagger$ Non-service connected Veterans determined by the VA to be low-income.

§Non-service connected Veterans who have income above the VA administered means test.

Hispanic was not associated with higher odds of a positive food insecurity (aOR $=1.00,95 \% \mathrm{CI}=0.89,1 \cdot 12)$. Additionally, while PTSD among women trended towards higher odds of a positive screen in adjusted analyses, this did not reach statistical significance $(\mathrm{aOR}=1.05,95 \%$ $\mathrm{CI}=0.99,1.13$ ) (Table 3). Adjusted and unadjusted odd ratios for correlates of a positive food insecurity clinical reminder for the overall cohort were similar to correlates among male Veterans given that $91 \%$ of the cohort was male (see online supplementary material, Supplemental Table 1).

Sensitivity analyses excluding a 6-month 'ramp-up' period from July 2017 to December 2017 did not yield substantively different findings relative to results from analyses using the full July 2017-December 2018 period.

\section{Discussion}

This study is, to our knowledge, the first to examine prevalence and risk factors associated with US Veteran food insecurity as identified in a nationwide VA food insecurity screener. More than 3.3 million non-institutionalised Veterans were screened for food insecurity in the first 18 months following implementation of the VA food insecurity clinical reminder. More than 44000 Veterans were identified as food insecure on their initial screen, representing $1.3 \%$ of men and $2.0 \%$ of women. While measures of lower income and financial hardship were associated with a positive food insecurity clinical reminder screen, a positive screen was also associated with numerous medical and trauma-related comorbidities such as MST. Veterans of 
Table 2 Characteristics of Veterans screened using the food insecurity clinical reminder, by screening status: July 2017-December 2018

\begin{tabular}{|c|c|c|c|c|c|c|c|c|c|c|c|c|}
\hline \multirow[b]{3}{*}{ Characteristics, $n(\%)$} & \multicolumn{4}{|c|}{ All Veterans } & \multicolumn{4}{|c|}{ Male Veterans } & \multicolumn{4}{|c|}{ Female Veterans } \\
\hline & \multicolumn{2}{|c|}{$\begin{array}{l}\text { Positive } \\
\text { screen } \\
\text { (n } 44298)\end{array}$} & \multicolumn{2}{|c|}{$\begin{array}{l}\text { Negative screen } \\
\text { (n } 3260404)\end{array}$} & \multicolumn{2}{|c|}{$\begin{array}{l}\text { Positive } \\
\text { screen } \\
\text { (n } 38 \text { 449) }\end{array}$} & \multicolumn{2}{|c|}{$\begin{array}{l}\text { Negative screen } \\
\text { (n2 } 967348)\end{array}$} & \multicolumn{2}{|c|}{$\begin{array}{l}\text { Positive } \\
\text { screen } \\
(n \text { 5849) }\end{array}$} & \multicolumn{2}{|c|}{$\begin{array}{c}\text { Negative } \\
\text { screen } \\
(n 293 \text { 056) }\end{array}$} \\
\hline & $n$ & $\%$ & $n$ & $\%$ & $n$ & $\%$ & $n$ & $\%$ & $n$ & $\%$ & $n$ & $\%$ \\
\hline \multicolumn{13}{|l|}{ Age } \\
\hline $18-34$ & 6582 & 14.9 & 321334 & $9 \cdot 9$ & 5186 & $13 \cdot 5$ & 257017 & $8 \cdot 7$ & 1396 & 23.9 & 64317 & 21.9 \\
\hline $35-44$ & 5885 & $13 \cdot 3$ & 303526 & $9 \cdot 3$ & 4512 & $11 \cdot 7$ & 242689 & $8 \cdot 2$ & 1373 & $23 \cdot 5$ & 60837 & $20 \cdot 8$ \\
\hline $45-54$ & 7396 & $16 \cdot 7$ & 407187 & $12 \cdot 5$ & 6103 & $15 \cdot 9$ & 344416 & $11 \cdot 6$ & 1293 & $22 \cdot 1$ & 62771 & 21.4 \\
\hline $55-64$ & 14631 & $33 \cdot 0$ & 601805 & $18 \cdot 5$ & 13217 & 34.4 & 533118 & $18 \cdot 0$ & 1414 & $24 \cdot 2$ & 68687 & 23.4 \\
\hline$\geq 65$ & 9804 & $22 \cdot 1$ & 1626552 & $49 \cdot 9$ & 9431 & 24.5 & 1590108 & $53 \cdot 6$ & 373 & $6 \cdot 4$ & 36444 & $12 \cdot 4$ \\
\hline \multicolumn{13}{|l|}{ Race } \\
\hline White, non-Hispanic & 21769 & $49 \cdot 1$ & 2176260 & $66 \cdot 7$ & 19278 & $50 \cdot 1$ & 2024546 & $68 \cdot 2$ & 2491 & $42 \cdot 6$ & 151714 & $51 \cdot 8$ \\
\hline Black, non-Hispanic & 14567 & $32 \cdot 9$ & 558824 & $17 \cdot 1$ & 12215 & $31 \cdot 8$ & 470805 & $15 \cdot 9$ & 2352 & $40 \cdot 2$ & 88019 & $30 \cdot 0$ \\
\hline Hispanic & 3898 & $8 \cdot 8$ & 212136 & 6.5 & 3476 & $9 \cdot 0$ & 189085 & $6 \cdot 4$ & 422 & $7 \cdot 2$ & 23051 & 7.9 \\
\hline Other, non-Hispanic & 2022 & $4 \cdot 6$ & 134769 & $4 \cdot 1$ & 1700 & 4.4 & 118670 & $4 \cdot 0$ & 322 & $5 \cdot 5$ & 16099 & $5 \cdot 5$ \\
\hline Missing & 2604 & 5.9 & 213955 & $6 \cdot 6$ & 2258 & 5.9 & 195557 & $6 \cdot 6$ & 346 & 5.9 & 18398 & $6 \cdot 3$ \\
\hline \multicolumn{13}{|l|}{ Marital status } \\
\hline Married/partnered & 11232 & 25.4 & 1737794 & $53 \cdot 3$ & 10091 & $26 \cdot 2$ & 1633529 & $55 \cdot 1$ & 1141 & $19 \cdot 5$ & 104265 & $35 \cdot 6$ \\
\hline Divorced/separated/widowed & 20111 & $45 \cdot 4$ & 929046 & 28.5 & 17215 & $44 \cdot 8$ & 817840 & $27 \cdot 6$ & 2896 & $49 \cdot 5$ & 111206 & 37.9 \\
\hline Single, never married & 10977 & $24 \cdot 8$ & 430926 & $13 \cdot 2$ & 9353 & $24 \cdot 3$ & 364736 & $12 \cdot 3$ & 1624 & $27 \cdot 8$ & 66190 & $22 \cdot 6$ \\
\hline Missing & 1978 & 4.5 & 162638 & $5 \cdot 0$ & 1790 & $4 \cdot 7$ & 151243 & $5 \cdot 1$ & 188 & $3 \cdot 2$ & 11395 & 3.9 \\
\hline Rural & 9936 & $22 \cdot 4$ & 1096596 & 33.6 & 8815 & $22 \cdot 9$ & 1022421 & 34.5 & 1121 & $19 \cdot 2$ & 74175 & $25 \cdot 3$ \\
\hline \multicolumn{13}{|l|}{ Enrolment priority* } \\
\hline SC disability† & 20559 & $46 \cdot 4$ & 1818415 & $55 \cdot 8$ & 17143 & $44 \cdot 6$ & 1624003 & $54 \cdot 7$ & 3416 & $58 \cdot 4$ & 194412 & $66 \cdot 3$ \\
\hline Non-SC and low-incomeł & 19519 & $44 \cdot 1$ & 715649 & 21.9 & 17556 & $45 \cdot 7$ & 659620 & $22 \cdot 2$ & 1963 & $33 \cdot 6$ & 56029 & $19 \cdot 1$ \\
\hline Non-SC and not low-income§ & 3961 & $8 \cdot 9$ & 715790 & $22 \cdot 0$ & 3547 & $9 \cdot 2$ & 675069 & $22 \cdot 7$ & 414 & $7 \cdot 1$ & 40721 & 13.9 \\
\hline Missing & 259 & 0.6 & 10550 & 0.3 & 203 & $0 . \overline{5}$ & 8656 & 0.3 & 56 & 1.0 & 1894 & 0.6 \\
\hline Substance use disorder & 12809 & 28.9 & 286421 & $8 \cdot 8$ & 11710 & 30.5 & 267529 & $9 \cdot 0$ & 1099 & $18 \cdot 8$ & 18892 & $6 \cdot 4$ \\
\hline Current smoker & 21392 & $48 \cdot 3$ & 774611 & $23 \cdot 8$ & 19104 & $49 \cdot 7$ & 715722 & $24 \cdot 1$ & 2288 & $39 \cdot 1$ & 58889 & $20 \cdot 1$ \\
\hline $\begin{array}{l}\text { Homelessness/housing instability } \\
\text { BMI }\end{array}$ & 25751 & $58 \cdot 1$ & 178228 & $5 \cdot 5$ & 22503 & $58 \cdot 5$ & 156832 & $5 \cdot 3$ & 3248 & $55 \cdot 5$ & 21396 & $7 \cdot 3$ \\
\hline$<18.5$ & 855 & 1.9 & 25587 & 0.8 & 728 & 1.9 & 22491 & 0.8 & 127 & $2 \cdot 2$ & 3096 & $1 \cdot 1$ \\
\hline $18 \cdot 5-24.9$ & 11226 & $25 \cdot 3$ & 541535 & $16 \cdot 6$ & 9974 & $25 \cdot 9$ & 483003 & $16 \cdot 3$ & 1252 & 21.4 & 58532 & $20 \cdot 0$ \\
\hline $25-29 \cdot 9$ & 12594 & 28.4 & 1063487 & $32 \cdot 6$ & 11183 & $29 \cdot 1$ & 982855 & $33 \cdot 1$ & 1411 & $24 \cdot 1$ & 80632 & 27.5 \\
\hline $30-34.9$ & 8311 & $18 \cdot 8$ & 796238 & 24.4 & 7085 & $18 \cdot 4$ & 728888 & $24 \cdot 6$ & 1226 & $21 \cdot 0$ & 67350 & $23 \cdot 0$ \\
\hline$\geq 35$ & 6216 & $14 \cdot 0$ & 522421 & $16 \cdot 0$ & 5029 & $13 \cdot 1$ & 465090 & $15 \cdot 7$ & 1187 & $20 \cdot 3$ & 57331 & $19 \cdot 6$ \\
\hline Missing & 5096 & 11.5 & 311136 & 9.5 & 4450 & $11 \cdot 6$ & 285021 & $9 \cdot 6$ & 646 & $11 \cdot 0$ & 26115 & 8.9 \\
\hline Diabetes & 8935 & $20 \cdot 2$ & 798975 & $24 \cdot 5$ & 8170 & $21 \cdot 2$ & 765523 & $25 \cdot 8$ & 765 & $13 \cdot 1$ & 33452 & 11.4 \\
\hline Hypertension & 18141 & $41 \cdot 0$ & 1707087 & $52 \cdot 4$ & 16538 & $43 \cdot 0$ & 1624887 & $54 \cdot 8$ & 1603 & $27 \cdot 4$ & 82200 & $28 \cdot 0$ \\
\hline Depression & 16027 & $36 \cdot 2$ & 545218 & $16 \cdot 7$ & 13254 & 34.5 & 456217 & $15 \cdot 4$ & 2773 & 47.4 & 89001 & $30 \cdot 4$ \\
\hline Post-traumatic stress disorder & 10547 & $23 \cdot 8$ & 478844 & $14 \cdot 7$ & 8484 & $22 \cdot 1$ & 417133 & $14 \cdot 1$ & 2063 & $35 \cdot 3$ & 61711 & $21 \cdot 1$ \\
\hline History of military sexual trauma & 5146 & $11 \cdot 6$ & 143531 & $4 \cdot 4$ & 2288 & $6 \cdot 0$ & 53448 & $1 \cdot 8$ & 2858 & $48 \cdot 9$ & 90083 & $30 \cdot 7$ \\
\hline
\end{tabular}

Abbreviations: SC, service connected; VA, US Department of Veterans Affairs; BMI, body mass index.

*Enrolment priority determines US Veterans' eligibility for, and cost-share associated with, VA health benefits.

†SC disability provides a monetary benefit paid to Veterans who are determined by VA to be disabled by an injury or illness that was incurred or aggravated during active military service.

\#Non-service connected Veterans determined by the VA to be low-income.

$\S$ Non-service connected Veterans who have income above the VA administered means test.

colour and women were also at increased risk for a positive food insecurity screen.

The prevalence of Veteran food insecurity identified by the VA food insecurity clinical reminder is markedly lower than rates found in prior studies ${ }^{(3,5,17-19)}$. This may reflect differences in the single-item question used in the VA screener compared with those used in prior research $^{(3,5,17,18,21)}$. For example, the gold standard USDA Food Security Survey Module includes questions such as being 'worried' whether food would run out before there was money to buy more ${ }^{(24)}$. In contrast, the VA food insecurity clinical reminder asks whether a Veteran has 'run out of food,' a more severe form of food hardship. Additionally, the food insecurity clinical reminder provides binary 'yes/ no' response options, which have been shown to identify substantially less need than offering 'often/sometimes/ never' categories ${ }^{(25)}$. For these reasons, it is likely that the VA screener was capturing only the most severe cases of food insecurity among Veterans. Reported rates of food insecurity may also have been lower in our sample compared with survey-based estimates due to well-recognised barriers to disclosure of food insecurity in clinical settings including stigma, fear of judgment and concern about being reported to child protective services ${ }^{(26-29)}$. As with other food insecurity instruments commonly used both in clinical settings and in survey research ${ }^{(24,30)}$, the VA 
Table 3 Correlates of a positive food insecurity clinical reminder screen, by gender

\begin{tabular}{|c|c|c|c|c|c|c|c|c|}
\hline \multirow[b]{2}{*}{ Characteristics } & \multicolumn{4}{|c|}{ Male Veterans } & \multicolumn{4}{|c|}{ Female Veterans } \\
\hline & OR & $95 \% \mathrm{Cl}$ & $\mathrm{aOR}$ & $95 \% \mathrm{Cl}$ & OR & $95 \% \mathrm{Cl}$ & $\mathrm{aOR}$ & $95 \% \mathrm{Cl}$ \\
\hline \multicolumn{9}{|l|}{ Age } \\
\hline $18-34$ & 1.64 & $1.55,1.75$ & 1.93 & $1 \cdot 71,2 \cdot 18$ & 1.13 & $1.05,1.21$ & 1.69 & $1.48,1.92$ \\
\hline $35-44$ & 1.50 & $1.41,1.58$ & 1.87 & $1.68,2.08$ & 1.17 & $1 \cdot 10,1 \cdot 24$ & 1.72 & $1.52,1.95$ \\
\hline $45-54$ & 1.44 & $1.36,1.52$ & 1.66 & $1.51,1.82$ & 1.03 & $0.96,1.11$ & 1.51 & $1.35,1.69$ \\
\hline $55-64$ & $2 \cdot 40$ & $2 \cdot 24,2 \cdot 56$ & 1.58 & $1 \cdot 44,1 \cdot 73$ & 1.04 & $0.97,1.12$ & $1 \cdot 31$ & $1 \cdot 16,1.48$ \\
\hline \multicolumn{9}{|l|}{ Race/ethnicity } \\
\hline White, Non-Hispanic & ref & & ref & & ref & & ref & \\
\hline Black, Non-Hispanic & $2 \cdot 47$ & $2 \cdot 24,2 \cdot 72$ & 1.32 & $1.25,1.40$ & 1.56 & $1.45,1.69$ & 1.24 & $1 \cdot 16,1 \cdot 33$ \\
\hline Hispanic & 1.47 & $1.17,1.85$ & 1.48 & $1.06,2.08$ & 0.91 & $0.80,1.03$ & 1.00 & $0.89,1.12$ \\
\hline Other, Non-Hispanic & $1 \cdot 11$ & $0.99,1.25$ & $1 \cdot 22$ & $1 \cdot 12,1 \cdot 33$ & 1.01 & $0.89,1 \cdot 15$ & $1 \cdot 14$ & $1 \cdot 00,1 \cdot 29$ \\
\hline Missing & 0.88 & $0.81,0.96$ & 1.07 & $0.96,1.19$ & 0.93 & $0.83,1.05$ & 1.21 & $1.07,1.36$ \\
\hline \multicolumn{9}{|l|}{ Marital Status } \\
\hline Married/partnered & ref & & ref & & ref & & ref & \\
\hline Non-married/partnered & $3 \cdot 38$ & $3.06,3.73$ & 1.36 & $1.30,1.42$ & $2 \cdot 23$ & $2 \cdot 10,2 \cdot 37$ & 1.36 & $1.27,1.44$ \\
\hline Missing & 0.91 & $0.79,1.05$ & $1 \cdot 27$ & $1 \cdot 10,1 \cdot 46$ & 0.81 & $0.69,0.94$ & $1 \cdot 26$ & $1.08,1.47$ \\
\hline Rural & 0.57 & $0.50,0.65$ & 1.02 & $0.91,1.15$ & 0.70 & $0.63,0.78$ & 0.99 & $0.91,1.09$ \\
\hline \multicolumn{9}{|l|}{ Enrolment priority status* } \\
\hline SC Disability† & ref & & ref & & ref & & ref & \\
\hline Non-SC and low-income & $2 \cdot 96$ & $2 \cdot 80,3 \cdot 13$ & 1.52 & $1.47,1.58$ & $2 \cdot 15$ & $2 \cdot 02,2 \cdot 29$ & 1.52 & $1.43,1.62$ \\
\hline Non-SC and not low-income§ & 0.35 & $0.31,0.38$ & 0.85 & $0.79,0.91$ & 0.47 & $0.42,0.53$ & 0.93 & $0.83,1.05$ \\
\hline Substance use disorder & 4.42 & $4 \cdot 10,4 \cdot 77$ & 0.97 & $0.93,1.02$ & 3.35 & $3 \cdot 11,3 \cdot 62$ & 1.05 & $0.96,1.14$ \\
\hline Current smoker & $3 \cdot 11$ & $2 \cdot 87,3 \cdot 38$ & $1 \cdot 35$ & $1 \cdot 30,1.41$ & 2.56 & $2 \cdot 40,2 \cdot 74$ & 1.53 & $1.42,1.63$ \\
\hline $\begin{array}{l}\text { Homelessness/housing instability } \\
\text { BMI }\end{array}$ & $25 \cdot 23$ & $22 \cdot 06,28 \cdot 85$ & $13 \cdot 79$ & $12 \cdot 36,15 \cdot 40$ & $15 \cdot 82$ & $14 \cdot 58,17 \cdot 16$ & $10 \cdot 70$ & $9 \cdot 77,11 \cdot 70$ \\
\hline $\begin{array}{l}<18.5 \\
18 \cdot 5-24.9\end{array}$ & $\begin{array}{l}2.52 \\
\text { ref }\end{array}$ & $2 \cdot 31,2 \cdot 75$ & $\begin{array}{l}1.31 \\
\text { ref }\end{array}$ & $1 \cdot 21,1 \cdot 41$ & $\begin{array}{l}2.08 \\
\text { ref }\end{array}$ & $1 \cdot 75,2 \cdot 47$ & $\begin{array}{l}1.53 \\
\text { ref }\end{array}$ & $1 \cdot 26,1 \cdot 87$ \\
\hline $25-29 \cdot 9$ & 0.83 & $0.80,0.86$ & 0.73 & $0.71,0.76$ & 0.84 & $0.79,0.89$ & 0.86 & $0.80,0.94$ \\
\hline $30-34.9$ & 0.69 & $0.67,0.72$ & 0.67 & $0.64,0.69$ & 0.89 & $0.83,0.96$ & 0.89 & $0.81,0.98$ \\
\hline$\geq 35$ & $0 \cdot 81$ & $0.77,0.85$ & 0.71 & $0.67,0.74$ & 1.05 & $0.98,1.12$ & 0.96 & $0.88,1.05$ \\
\hline Missing & 1.22 & $1.06,1.41$ & 0.90 & $0.83,0.98$ & $1 \cdot 25$ & $1.09,1.43$ & 1.07 & $0.96,1.20$ \\
\hline Diabetes & 0.78 & $0.73,0.83$ & $1 \cdot 13$ & $1 \cdot 09,1 \cdot 18$ & $1 \cdot 17$ & $1 \cdot 08,1 \cdot 27$ & $1 \cdot 22$ & $1 \cdot 11,1 \cdot 33$ \\
\hline Hypertension & 0.63 & $0.58,0.67$ & 0.84 & $0.80,0.88$ & 0.97 & $0.90,1.04$ & 0.93 & $0.87,1.00$ \\
\hline Depression & $2 \cdot 90$ & $2 \cdot 69,3 \cdot 12$ & 1.25 & $1 \cdot 20,1 \cdot 31$ & 2.07 & $1 \cdot 96,2 \cdot 19$ & $1 \cdot 22$ & $1 \cdot 15,1 \cdot 30$ \\
\hline Post-traumatic stress disorder & 1.73 & $1 \cdot 62,1 \cdot 85$ & 1.05 & $1.01,1.09$ & $2 \cdot 05$ & $1 \cdot 92,2 \cdot 19$ & 1.05 & $0.99,1.13$ \\
\hline History of military sexual trauma & 3.44 & $3 \cdot 19,3 \cdot 71$ & 1.42 & $1.35,1.50$ & $2 \cdot 15$ & $2 \cdot 01,2 \cdot 29$ & 1.49 & $1.40,1.59$ \\
\hline
\end{tabular}

Abbreviations: aOR, adjusted OR; SC, service connected; VA, US Department of Veterans Affairs, BMI, body mass index.

Multivariable logistic regression model, adjusted for VA Medical Center-level fixed effects.

*Enrolment priority determines Veterans' eligibility for, and cost-share associated with, VA health benefits.

†SC disability provides a monetary benefit paid to Veterans who are determined by VA to be disabled by an injury or illness that was incurred or aggravated during active military service.

¥Non-service connected Veterans determined by the VA to be low-income.

$\S$ Non-service connected Veterans who have income above the VA administered means test.

clinical reminder also focuses on financial barriers to food access and does not provide information on the nutritional adequacy of the food Veterans are able to obtain.

Similar to prior studies, we found that Veterans who were non-married/partnered ${ }^{(16,17,20,21)}$, low income ${ }^{(3,5,16,17,20)}$ or experienced housing instability within the past year ${ }^{(3,22)}$ were at increased risk for food insecurity. While the association between food insecurity and other markers of financial hardship such being low income or having unstable housing is unsurprising, findings of increased risk for food insecurity among those who are non-married/partnered may reflect having fewer financial reserves and potentially less social support to help stretch limited resources. Also consistent with other studies, we found that Veterans of colour were at increased risk of food insecurity ${ }^{(3,17)}$. This increased risk likely reflects a number of complex and interrelated factors including a disproportionate burden of poverty and unemployment resulting from structural racism and disparities in social and economic opportunity ${ }^{(14,31)}$, as well as experiences of racism and racial and ethnic discrimination ${ }^{(32,33)}$.

Also consistent with prior studies, we found that Veterans with a history of depression and/or PTSD had higher odds of a positive food insecurity screen ${ }^{(3,5,17,20)}$. Veterans with diabetes also had higher odds of food insecurity, which can impede pharmaceutical-based efforts to ensure adequate control of this diet-related disease. This is consistent with prior work finding poorer diabetes control among both Veterans and non-Veterans experiencing food insecurity ${ }^{(3,10,34)}$. Although US households in rural areas experience food insecurity at rates significantly higher than the national average ${ }^{(9)}$, we did not find an association between living in a rural area and food insecurity. One reason for this may be that because the clinical 
reminder question focuses on financial barriers to obtaining food, the screener does not explicitly assess other barriers to food access that may be more salient in rural areas such as limited food availability or lack of transportation.

Findings from our study and others ${ }^{(17,20,21)}$ that Veterans under 65 were more likely to screen positive for food insecurity likely reflects multiple factors. Volunteer-era (i.e. post-Vietnam) Veterans have both poorer financial stability and higher levels of material hardship than their counterparts from earlier service periods ${ }^{(17,18,21,35)}$. Volunteer-era Veterans are more likely to come from a lower socioeconomic background and have lower educational attainment ${ }^{(36,37)}$, and are more likely to report a history of childhood or other trauma prior to military service ${ }^{(38)}$. Each of these factors has been associated with higher risk of food insecurity later in life $e^{(39,40)}$. Furthermore, Veterans age 65 and older may be more likely to have resources to promote financial stability compared to Veterans under age 65 . These resources may include social security retirement benefits, receipt of a military pension or retirement savings from a civilian job.

Veterans with some degree of service-connected disability - and who therefore also receive VA disabilityrelated compensation-had lower odds of a positive food insecurity screen than low-income Veterans without a service-connected disability. This parallels findings of Montgomery and colleagues pertaining to serviceconnected disability and Veterans' risk of homelessness and housing instability ${ }^{(41)}$. Together, these findings suggest that VA disability compensation provides some measure of protection from material hardship. Future work is needed to better understand how this protective effect may vary by type and severity of serviceconnected disability as well as benefit amount. Findings also suggest that Veterans who are low-income and not receiving VA disability-related compensation may benefit from additional targeted food insecurity screening efforts.

Our study is the first to have specifically examined the association between MST and food insecurity. There is, however, a well-established association between food insecurity and past and/or current trauma such as sexual or physical violence ${ }^{(42)}$ as well as history of adverse childhood experiences ${ }^{(40)}$. Prior studies have also found an association between history of MST and post-deployment homelessness ${ }^{(43,44)}$. These relationships are likely multifactorial and reflect the association between trauma and medical and mental health-related comorbidities, as well as higher rates of unemployment, financial hardship and decreased self-management capacity. Similar to our findings that history of MST was independently associated with food insecurity, Brignone and colleagues found that MST was independently associated with homelessness even after adjusting for co-occurring mental health conditions ${ }^{(44)}$. Taken together, these findings underscore the complex interplay between trauma exposure and subsequent material hardship.

History of MST was associated an increased odds of food insecurity among both men and women. Woman overall, however, were nearly 17 times more likely than men to have experienced MST (31.1\% of women $v .1 .9 \%$ of men), which is consistent with previously reported rates of $\mathrm{MST}^{(45)}$. Among women screening positive for food insecurity, nearly half (48.9\%) reported a history of MST. Compared with men, women overall also had twice the rate of depression, $50 \%$ higher rates of PTSD and as in prior studies they were also more likely to have experienced recent homelessness or housing instability ${ }^{(41,46)}$, all of which were associated with increased risk for food insecurity. Each of these factors highlights the unique and complex challenges commonly faced by women Veterans.

Collectively, these findings can help clinicians focus additional targeted screening efforts which may include both more frequent screening among populations at particularly high risk for food insecurity, and also expansion of routine screening beyond primary care to include settings such as mental health clinics. Identifying Veterans vulnerable to food insecurity is particularly urgent given the current COVID-19 pandemic, which has both exacerbated existing disparities around food access and plunged many who were previously food secure into new material hardship $^{(15)}$. In some settings such as VA homeless clinics, it may be appropriate to screen for food insecurity at every visit. The strong association independent between food insecurity and both MST and PTSD underscores the need for trauma informed care regardless of screening setting.

Our findings can also help providers and health care organisations prioritise the development of wrap-around, team-based interventions tailored to specific high-risk groups. Given that Veterans with diabetes and mental health-related comorbidities are at increased risk for food insecurity, targeted trainings regarding risks for and sequalae of food insecurity may be indicated for those providing clinical care for these populations including primary care and mental health providers, endocrinologists, pharmacists and dietitians. Trainings should cover the need to review medication lists with patients for any cost-related barriers to adherence, medications with high risk for hypoglycaemia or medications requiring specific food availability, as well as the importance of providing context-appropriate nutritional counselling based on patients' medical and social circumstances. Interventions to ameliorate food insecurity may include development and/or expansion of onsite food pantries, referrals to community-based emergency food resources to meet immediate needs, onsite support applying for federal food assistance programs and/or direct provision of food through produce prescriptions or medically tailored meal programs. VA facilities may also partner with Veteran Service Organizations to facilitate connecting Veterans with available community and governmental resources. 
The substantial variation in rates of positive screens across individual VA medical facilities likely reflects several factors including both geographical variation in the community-level prevalence of food insecurity as well as facility-level differences in screening practices and populations of Veterans served. Although the VA food insecurity clinical reminder question and follow-up prompts are uniform across VA medical centres, there is local variation in who administers the screening (e.g. clinician, nurse, medical assistant, social worker, dietitian) and whether the screener is administered prior to seeing a provider or during the clinical encounter. Additional research is needed to explore variation in how - and how reliably_screening is administered, as well as how variation in screening administration may impact Veterans' responses. Prior work has found patients prefer self-administered paper or tabletbased food insecurity screening rather than being screened verbally ${ }^{(26,47,48)}$, and that disclosure rates for food insecurity in clinical settings are higher when screening is self-administered $^{(47-49)}$. While the VA food insecurity clinical reminder — similar to other VA clinical reminders-is currently designed to be verbally administered by a member of the clinical care team, considering potential mechanisms for self-administered screening may be warranted.

Future work should examine variation at both the individual and medical centre level in how Veterans' needs are addressed once they are identified as food insecure. There is also a need to better understand how Veterans' experiences of food insecurity and food insecurity clinical reminder screening results may change over time, as well as optimal intervals for rescreening and whether this should vary by population. Finally, in April of 2021, which postdated our study period, VHA updated the food insecurity clinical reminder to use a two-question instrument that has been validated to assess risk for food insecurity in clinical settings ${ }^{(30)}$. Future work should explore how the prevalence of food insecurity identified within VHA may change with this new instrument.

\section{Limitations}

Our study has several limitations. First, because the VA food insecurity clinical reminder asks about particularly severe food hardship it may be underestimating the true prevalence of food insecurity among Veterans receiving VA care. While future validation studies are needed to understand how findings from the VA screener compare with other food insecurity instruments, it seems likely that those Veterans identified by the VA clinical reminder are at particularly high risk for having immediate food needs. Second, we were only able to analyse data for Veterans who presented for care within VHA and were screened during the first 18 months following implementation of the food insecurity clinical reminder. In particular, we were unable to examine food security status for those Veterans who did not engage in VHA care during the 18-month study period either because they did not seek healthcare during this period, they sought care outside of the VA using other benefits such as Medicare or Medicaid and/or they sought care in the community through the Choice $\mathrm{Act}^{(50)}$. A unique strength of this study is we were able to use a national administrative VA database to evaluate the entire population of 3.3 million Veterans screened. Third, there is local variation in how screening is administered which could impact Veterans' response. We did, however, include VA medical centre-level fixed effects in our models to account for stable facility-level differences in screening practices and populations. Fourth, responses may have been influenced by perceived stigma, social-desirability bias and Veterans' comfort with or trust in the person administering the screening. Results may have varied with a self-administered screener ${ }^{(49)}$.

\section{Conclusions}

Systematic universal screening for food insecurity in the VA is a critical first step towards identifying Veterans currently experiencing or at high risk for experiencing food insecurity. Future work is needed to identify best practices for connecting Veterans experiencing food insecurity with VA and community resources to most effectively address the unique needs of the Veteran population.

\section{Acknowledgements}

Acknowledgements: We thank Robert McConeghy for his assistance with early data analyses, as well as Jason Buxbaum for his thoughtful review of the manuscript. Financial support: This work was supported by grant CIN 13-419 from the VA Health Services Research and Development Center of Innovation in Long Term Services and Supports. A.J.C. was additionally supported by CDA 20-037 from the Department of Veterans Affairs Health Services Research and Development. M.H. was supported by grant P30DK092926 (MCDTR) from the National Institute of Diabetes and Digestive and Kidney Diseases. K.S.T was supported by CDA 14-422 from the Department of Veterans Affairs Health Services Research and Development. The funders had no role in the design and conduct of the study; collection, management, analysis and interpretation of the data; preparation, review or approval of the manuscript or the decision to submit the manuscript for publication. The manuscript's contents are solely the responsibility of the authors and do not reflect the position or policy of the Department of Veterans Affairs, the US government, the National Institutes of Health, or other affiliated institutions. Conflict of interest: There are no conflicts of interest. Authorship: Study concept and design: A.J.C., D.M.D., J.L.R., M.H., K.S.T. Acquisition, 
analysis or interpretation of data: all authors. Drafting of the manuscript: A.J.C. Critical revision of the manuscript for important intellectual content: all authors. Statistical analysis: C.W.H., A.J.C. Obtaining funding: J.L.R., A.J.C. Administrative, technical, or material support and supervision: A.J.C., D.M.D., J.L.R., K.S.T. Final approval of the version to be published: all authors. Ethics of human subject participation: This study was conducted according to the guidelines laid down in the Declaration of Helsinki and all procedures involving research study participants were approved by the Providence VA Medical Center IRB, including a waiver of informed consent as analyses were limited to retrospective chart review.

\section{Supplementary material}

For supplementary material/s referred to in this article, please visit https://doi.org/10.1017/S1368980021004584

\section{References}

1. USDA ERS (2021) Food Insecurity in the US - Measurement. https://www.ers.usda.gov/topics/food-nutrition-assistance/ food-security-in-the-us/measurement/\#security （accessed September 2021).

2. Gundersen C \& Ziliak JP (2015) Food insecurity and health outcomes. Health Aff Proj Hope 34, 1830-1839.

3. Wang EA, McGinnis KA, Goulet J et al. (2015) Food insecurity and health: data from the veterans aging cohort study. Public Health Rep 130, 261-268.

4. Kushel MB, Gupta R, Gee L et al. (2006) Housing instability and food insecurity as barriers to health care among lowincome Americans. J Gen Intern Med 21, 71-77.

5. Narain K, Bean-Mayberry B, Washington DL et al. (2018) Access to care and health outcomes among women veterans using veterans administration health care: association with food insufficiency. Womens Health Issues 28, 267-272.

6. Dean EB, French MT \& Mortensen K (2020) Food insecurity, health care utilization, and health care expenditures. Health Serv Res 55, 883-893.

7. Berkowitz SA, Seligman HK, Meigs JB et al. (2018) Food insecurity, healthcare utilization, and high cost: a longitudinal cohort study. Am J Manag Care 24, 399-404.

8. Berkowitz SA, Basu S, Meigs JB et al. (2017) Food insecurity, health care expenditures in the United States, 2011-2013. Health Serv Res 53, 1600-1620.

9. Coleman-Jensen A, Rabbitt MP, Gregory C et al. (2021) Household Food Security in the United States in 2020. http://www.ers.usda.gov/publications/pub-details/?pubid= 102075 (accessed October 2021).

10. Berkowitz SA, Meigs JB, DeWalt D et al. (2015) Material need insecurities, control of diabetes mellitus, and use of health care resources: results of the measuring economic insecurity in diabetes study. JAMA Intern Med 175, 257-265.

11. Berkowitz SA, Seligman HK \& Choudhry NK (2014) Treat or Eat: food Insecurity, cost-related Medication Underuse, and Unmet Needs. Am J Med 127, 310.e3.

12. McDougall JA, Anderson J, Adler Jaffe S et al. (2020) Food insecurity and forgone medical care among cancer survivors. JCO Oncol Pract 16, e922-e932.

13. Restrepo BJ, Rabbitt MP \& Gregory CA (2021) The effect of unemployment on food spending and adequacy: evidence from coronavirus-induced firm closures. Appl Econ Perspect Policy 43, 185-204.

14. Morales DX, Morales SA \& Beltran TF (2020) Racial/Ethnic disparities in household food insecurity during the COVID19 pandemic: a nationally representative study. J Racial Ethn Health Disparities 8, 1300-1314.

15. Wolfson JA \& Leung CW (2020) Food insecurity during COVID-19: an acute crisis with long-term health implications. Am J Public Health 110, 1763-1765.

16. Rabbitt MP \& Smith MD (2021) Food Insecurity among Working-Age Veterans. Washington, DC: USDA.

17. Pooler J, Mian P, Srinivasan M et al. (2018) Veterans and Food Insecurity. https://www.impaqint.com/sites/default/ files/issue-briefs/VeteransFoodInsecurity_IssueBrief_V1.3. pdf (accessed November 2018).

18. Miller DP, Larson MJ, Byrne T et al. (2016) Food insecurity in veteran households: findings from nationally representative data. Public Health Nutr 19, 1731-1740.

19. Schure MB, Katon JG, Wong E et al. (2016) Food and housing insecurity and health status among U.S. adults with and without prior military service. SSM - Popul Health 2, 244-248.

20. Brostow DP, Gunzburger E \& Thomas KS (2017) Food insecurity among veterans: findings from the health and retirement study. J Nutr Health Aging 21, 1358-1364.

21. Widome R, Jensen A, Bangerter A et al. (2015) Food insecurity among veterans of the US wars in Iraq and Afghanistan. Public Health Nutr 18, 844-849.

22. O'Toole TP, Roberts CB \& Johnson EE (2017) Screening for food insecurity in six veterans administration clinics for the homeless, June-December 2015. Prev Chronic Dis 14, E04.

23. Cohen AJ, Rudolph JL, Thomas KS et al. (2020) Food insecurity among veterans: resources to screen and intervene. Fed Pract 37, 16-23.

24. U.S. Department of Agriculture, Economic Research Service (2021) USDA ERS - Survey Tools. https://www.ers.usda. gov/topics/food-nutrition-assistance/food-security-in-theus/survey-tools/ (accessed October 2021).

25. Makelarski JA, Abramsohn E, Benjamin JH et al. (2017) Diagnostic accuracy of two food insecurity screeners recommended for use in health care settings. Am J Public Health 107, 1812-1817.

26. Cullen D, Attridge M \& Fein JA (2020) Food for thought: a qualitative evaluation of caregiver preferences for food insecurity screening and resource referral. Acad Pediatr 20, $1157-1162$.

27. Barnidge E, LaBarge G, Krupsky K et al. (2017) Screening for Food Insecurity in Pediatric Clinical Settings: opportunities and Barriers. J Community Health 42, 51-57.

28. Barnidge E, Krupsky K, LaBarge G et al. (2020) Food insecurity screening in pediatric clinical settings: a caregivers' perspective. Matern Child Health J 24, 101-109.

29. Palakshappa D, Doupnik S, Vasan A et al. (2017) Suburban families' experience with food insecurity screening in primary care practices. Pediatrics 140, e20170320.

30. Hager ER, Quigg AM, Black MM et al. (2010) Development and validity of a 2 -item screen to identify families at risk for food insecurity. Pediatrics 126, e26-32.

31. Odoms-Young A \& Bruce MA (2018) Examining the impact of structural racism on food insecurity: implications for addressing racial/ethnic disparities. Fam Community Health 41, S3-S6.

32. Phojanakong P, Brown WE, Grimaldi G et al. (2019) Experiences of racial and ethnic discrimination are associated with food insecurity and poor health. Int J Environ Res Public Health 16, 4369.

33. Burke MP, Jones SJ, Frongillo EA et al. (2018) Severity of household food insecurity and lifetime racial discrimination among African-American households in South Carolina. Ethn Health 23, 276-292. 
34. Seligman HK, Jacobs EA, López A et al. (2012) Food insecurity and glycemic control among low-income patients with type 2 diabetes. Diabetes Care 35, 233-238.

35. Gustman AL, Steinmeier TL \& Tabatabai N (2016) Declining wealth and work among male veterans in the health and retirement study. J Retire $\mathbf{4}, 77-98$.

36. Cohen J, Warner RL \& Segal DR (1995) Military service and educational attainment in the all-volunteer force. Soc Sci $Q$ 76, $88-104$

37. Lutz A (2008) Who joins the military? A look at race, class, and immigration status. J Political Mil Sociol 36, 167-188.

38. Blosnich JR, Dichter ME, Cerulli C et al. (2014) Disparities in adverse childhood experiences among individuals with a history of military service. JAMA Psychiatr 71, 1041-1048.

39. Vogel C, Lewis D, Ntani G et al. (2017) The relationship between dietary quality and the local food environment differs according to level of educational attainment: a cross-sectional study. PLoS One 12, e0183700.

40. Chilton MM, Rabinowich JR \& Woolf NH (2014) Very low food security in the USA is linked with exposure to violence. Public Health Nutr 17, 73-82.

41. Montgomery AE, Dichter ME, Thomasson AM et al. (2015) Demographic characteristics associated with homelessness and risk among female and male veterans accessing VHA outpatient care. Womens Health Issues Publ Jacobs Inst Womens Health 25, 42-48.

42. Ricks JL, Cochran SD, Arah OA et al. (2016) Food insecurity and intimate partner violence against women: results from the California Women's Health Survey. Public Health Nutr 19, 914-923.

43. Pavao J, Turchik JA, Hyun JK et al. (2013) Military sexual trauma among homeless veterans. J Gen Intern Med 2, S536-541.

44. Brignone E, Gundlapalli AV, Blais RK et al. (2016) Differential risk for homelessness among us male and female veterans with a positive screen for military sexual trauma. JAMA Psychiatr 73, 582-589.

45. Kimerling R, Gima K, Smith MW et al. (2007) The veterans health administration and military sexual trauma. Am J Public Health 97, 2160-2166.

46. Peterson R, Gundlapalli AV, Metraux S et al. (2015) Identifying homelessness among veterans using VA administrative data: opportunities to expand detection criteria. PLoS One 10, e0132664.

47. Cullen D, Woodford A \& Fein J (2019) Food for thought: a randomized trial of food insecurity screening in the emergency department. Acad Pediatr 19, 646-651.

48. Palakshappa D, Goodpasture M, Albertini L et al. (2019) Written $v$. verbal food insecurity screening in one primary care clinic. Acad Pediatr 20, 203-207.

49. Gottlieb L, Hessler D, Long D et al. (2014) A randomized trial on screening for social determinants of health: the iscreen study. Pediatrics 134, e1611-1618.

50. US Department of Veteran Affairs (2014) Veterans access, choice and accountability act of ('choice act') fact sheet. https://www.va.gov/opa/choiceact/documents/choice-actsummary.pdf (accessed October 2021). 